\title{
THE STATUS OF THE DOUBLE POLARIZED DD-FUSION EXPERIMENT
}

\author{
P.V. Kravchenko*', V.D. Fotyev, K.A. Ivshin, E.N. Komarov, L.M. Kotchenda, O.L. \\ Kotchenda, P.A. Kravtsov, S.G. Sherman, A.N. Soloviev, I.N. Soloviev, V.A. Trofimov, \\ A.A. Vasilyev and M.E. Vznuzdaev \\ Petersburg Nuclear Physics Institute named by B.P.Konstantinov of National Research Centre \\ "Kurchatov Institute (PNPI)", 188300 Gatchina, Russia \\ E-mail: kravchenko_pvepnpi.nrcki.ru
}

\begin{abstract}
A double-polarized dd-fusion experiment (PolFusion, PNPI, Gatchina) has been proposed to investigate the reactions $d+d \rightarrow{ }^{3} \mathrm{He}+n$ and $d+d \rightarrow t+p$ in the energy range of 10-100 $\mathrm{keV}$. The possibility of using a vector and tensor polarized beam and target combination creates the opportunities to measure the asymmetry of the differential cross section and spin-correlation coefficients in the $d+{ }^{3} \mathrm{He}$ and $d+t$ reactions. Suggested measurements offer capabilities for determination of the quintet-state suppression factor for both reactions, one of the goals of PolFusion experiment program. A brief description of mathematical model, which allow to predict the observables, status of experimental data and first test measurements are given. Overview of the experimental setup, Monte Carlo simulation studies and details of future analysis are discussed.
\end{abstract}

23rd International Spin Physics Symposium - SPIN2018 -

10-14 September, 2018

Ferrara, Italy

\footnotetext{
* Speaker.

${ }^{\dagger}$ For PolFusion experiment.
} 


\section{Introduction}

Due to the limited supply of fossil fuels and to decrease the production of nuclear waste, thermonuclear fusion reactors are envisaged to replace the fission reactors besides the use of regenerative energy conversion. The principal aspects of the thermonuclear fusion problem include: (i) to understand the nuclear physics aspects of the fundamental fusion reactions at low energies, (ii) confinement of the hot and dense plasma, (iii) extraction of energy from plasma. Standard candidates for the fusion fuel are the hydrogen isotopes (the hydrogen, deuterium, tritium) and the light isotope of helium, ${ }^{3} \mathrm{He}$. As an input to the realization of the fusion reactors one needs the nuclear interaction cross sections of light nuclei. The same cross sections enter the analysis of energy production in stellar interior and the primordial nucleosynthesis at early stages of Big Bang. The cross sections of nuclear interactions of light nuclei have been extensively studied, but still not all of them are well known. This is especially true for the spin sector, although the spin effects in few-body reactions are large. Here we cite just three classical examples. (i) In the nucleon-nucleon system the bound state, the deuteron, exists only in the spin-triplet channel. The spin-singlet scattering length has a sign opposite to and about four times larger in magnitude than the spin-triplet scattering length. (ii) A strong resonance exists in the $n-{ }^{3} \mathrm{He}$ scattering cross section in the spin-singlet channel. The strong spin-singlet absorption enables an almost $100 \%$ spin filtering of neutrons with an excellent transmission rate of $50 \%$ with the use of a polarized $3 \mathrm{He}$ [1]. This technique has become one of the methods to generate intense polarized neutron beams [2]. (iii) The amplitudes of the important fusion reactions $\mathrm{D}+{ }^{3} \mathrm{H} \rightarrow n+{ }^{4} \mathrm{He}, \mathrm{D}+{ }^{3} \mathrm{He} \rightarrow p+{ }^{4} \mathrm{He}$ are dominated by the $\mathrm{S}$-wave $I^{P}=3 / 2^{+}$resonance. Such a resonance greatly simplifies the treatment of double-polarization effects: the numbers of the magnetic substates, four for $I=3 / 2$ and two for $I=1 / 2$, imply that in an unpolarized plasma only $2 / 3$ of nuclei undergo the fusion. Alternatively, a full polarization of the deuteron and ${ }^{3} \mathrm{He}$ would enhance the fusion cross section by $50 \%$. Such a strong polarization effect has been confirmed experimentally to a good accuracy [3]. Based on the scrutiny of the available experimental data on the d-d interaction, as early as in 1969 Ad'yasevich and Fomenko suggested the possibility of polarization enhancement of the DD fusion rate by a factor of two [4]. The first proposal by the Kurchatov Institute group on the experimental study of the polarization coefficients in double-polarized $\vec{d}(\vec{d}, p) t$ and $\vec{d}(\vec{d}, n)^{3} \mathrm{He}$ collisions in vacuum dates back to 1976 [5]. The proposal was not pursued as at that time polarized atomic and ion beams of adequate intensity were not available. A substantial step forward was made in 1982 in a theoretical study of the depolarization of nuclei in a magnetically confined plasma [6]. The principal conclusion was that the depolarization time greatly exceeds the fusion reaction time. Presently, it is considered feasible to confine ${ }^{3} \mathrm{He}$ with nuclear polarization reaching $55 \%$ and to inject neutral deuterium with nuclear polarization of about $55 \%$. The estimated enhancement of the fusion yield is $15 \%$. A corresponding experiment at the DIII-D tokamak at San Diego is planned [7]. In the case the polarization retention is confirmed, it is planned to look at the possibility of nuclear polarization of tritium, which would offer improved prospects of ignition in the ITER program and possible important cost savings. An alternative approach to the experimental confirmation of polarization persistence in a fusion process has been suggested at Orsay [8]: looking for nuclear reactions in a plasma generated by a petawatt laser hitting a polarized frozen HD target. Detecting the final state gammas and neutrons one would have an experimental access to polarization effects in the 
reactions $p+D \rightarrow{ }^{3} \mathrm{He}+\gamma$ and $D+D \rightarrow{ }^{3} \mathrm{He}+n$ by measuring the angular distributions relative to the quantization axis, the direction of the magnetic holding field.

\section{Polarization phenomena in few-body systems: theory and experiment}

A comprehensive review on the experimental data on polarization phenomena in few-body interactions of relevance to polarized fusion and of the theoretical approaches to the problem has recently been published [3]. As we already emphasized above, on the one hand the strong dominance of the $3 / 2^{+}$resonance mechanism tremendously simplifies the theoretical discussion of the single- and double-polarized $D-T$ and $D-{ }^{3} \mathrm{He}$ fusion. On the other hand, polarization effects in the $\mathrm{d}$-d fusion remain an entirely open issue.

When thinking of the theoretical approaches one must bear in mind that hitherto there are no direct experimental data on the fundamental neutron-neutron interaction and the existing experimental determinations of the neutron-neutron scattering length are contradictory. A consistent theory of three- and four-nucleon forces is as yet lacking, although a certain progress has been made in the framework of the effective field theories [9]. Referring to the review [3] for the details and references, we recall that tedious numerical solutions of the Faddev-Yakubovsky equations in the three-body p-d and n-d channels, based on the realistic potentials with allowance for the Coulomb interactions, do provide a satisfactory description of the experimental data. However, when it comes to a spin observable - the analyzing power $A_{y}$ - there is a persistent 20 to $30 \%$ disagreement between the theory and experiment. Such a disagreement extends to a four-body system. The calculations give a correct trend of the angular dependence of $A_{y}$ in elastic $p-{ }^{3} \mathrm{He}$ scattering, but the predicted analyzing power is by some $30 \%$ smaller that the experimental data. One tends to attribute these failures of the theory to poorly known three-nucleon forces. The existing database on polarized D-D interactions is restricted to the single-spin observables [3]. Again, the calculations do reproduce gross features of the angular dependence of the vector and tensor analyzing powers, but not their absolute values. Besides, the model dependence of the theoretical results exceeds the experimental errors. Of course, the theoretical analysis is greatly complicated by Coulomb effects, which are particularly strong at low energies - a complete solution of the Faddeev-Yakubovsky equations for the four-body channel is as yet lacking. In contrast to the $\mathrm{S}$-wave dominated $D-T$ fusion, a treatment of the $D-D$ fusion is further complicated by a large contribution from the $\mathrm{P}$ and $\mathrm{D}$ waves in the entrance channel, which persists even at very low energy of $3 \mathrm{keV}$. The presence of these partial waves suggests rather strong polarization correlations exceeding those in the polarized $D-T$ fusion.

On the theory side, one of the hopes for the progress is linked to possible incorporation of the nucleon-nucleon amplitudes from the effective field-theory analysis into the Resonating Group Method (RGM). An obvious advantage of the RGM, based on the variational approach, is that Coulomb effects can be incorporated much easier than in the Faddev-Yakubovsky technique. Here one recalls that in the three-body channel with the same input interaction the results from the RGM are consistent with the Faddeev-Yakubovsky results to one percent accuracy. A full merging of the RGM and of the effective field theory approach, a speciality of the theory group of the Institut für Kernphysik of Forschungszentrum Jülich, would require implementation of the supercomputer 
codes and could well take up to five years. The theoretical predictions then could be compared with the experimental data to be obtained for the polarization correlations in the $D-D$ interaction.

The reactions $\vec{d}+\vec{t}$ and $\vec{d}+{ }^{3} \vec{H} e$ with polarized initial particles have been fairly well investigated [3]. Spin-correlation components of the basic fusion reaction $\vec{d}+\vec{d}$, which is always present in the thermonuclear reactor with the energy range of $10-100 \mathrm{keV}$, are not measured experimentally yet. The two spin combinations, parallel and antiparallel deuteron-spin orientation, are important for the use of polarized fuel in the fusion reactor. Thermonuclear deuteron fusion reaction has the two channels: $\vec{d}+\vec{d} \rightarrow{ }^{3} \mathrm{He}+n$ and $\vec{d}+\vec{d} \rightarrow t+p$. Theoretical predictions for the ratio of the reaction cross section with parallel deuteron spin orientation $\sigma_{1,1}$ to the unpolarized cross section $\sigma_{0}$, defined as Quintet Suppression Factor (QSF), exhibit a very strong model dependence and are quite contradictory Fig. 1. Here $Q S F=1$ means that polarization of the initial particles does not affect the total reaction cross section. Fig. 1 features the results of calculations making use of various models. Significant scattering of the results illustrates a strong demand for the measurement of this ratio.

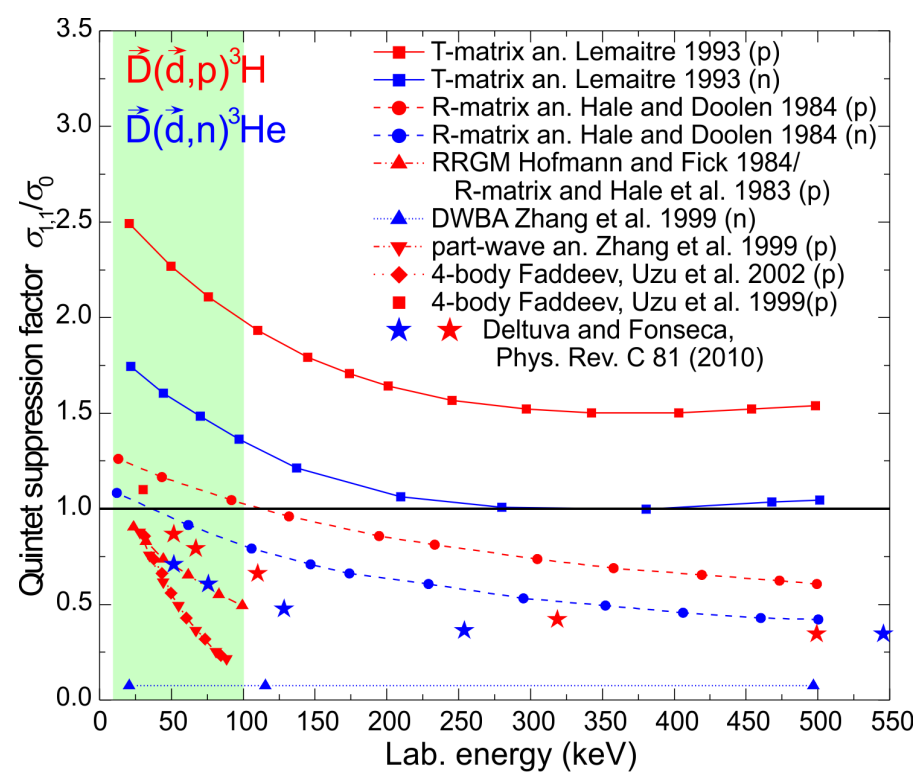

Figure 1: Theoretical predictions of the quintet suppression factor.

According to the variety of theoretical predictions, the planned measurements without polarization and with parallel deuteron spin orientation will yield answers to three questions.

- Is it possible to increase the D-D cross section by parallel deuteron spin orientation, possibly by a factor up to 2.5 ? This would raise the energy yield at the same input plasma density.

- Is it possible to modify the ratio of proton to neutron production favouring the first process? Neutrons are an unwelcome product of the fusion reactions in thermonuclear power reactors since they have large penetrating power. Therefore, possible suppression of the neutron channel is extremely desirable to reduce the radioactive waste and increase the lifetime of the blanket. 
- Is it possible to modify the angular distribution in the neutron emission by the use of polarized fuel? This is an important issue for the shielding of the reactor, since the neutron flux is one of the main factors destroying the reactor walls and blankets and it necessitates special shielding. Concentration of the neutron flux in predefined directions or planes helps to considerably reduce this danger and build the shield at dedicated locations.

The direct experimental measurement of the differential $D-D$ fusion cross section with parallel spin orientation may demonstrate the advantage to use polarized fuel in future fusion reactors. On the nuclear physics side, the principal objectives of the present program are the measurements of the asymmetry of differential cross sections, determination of the total cross-section change for the polarized initial particles, and measurement of the spin-correlation factors $C_{z, z}$ and $C_{z z, z z}$ for investigation of the quintet suppression factor.

\section{Experimental setup}

The spin-correlation coefficients for the interaction of polarized 10 to $100 \mathrm{keV}$ deuterons are to be determined by measuring the ejectile angular distributions of the reactions $\vec{d}+\vec{d} \rightarrow{ }^{3} \mathrm{He}+n$ and $\vec{d}+\vec{d} \rightarrow t+p$.

Due to a very low energy of the initial particles their penetrating power is exceedingly small less than a micrometer in a solid target. Therefore, the d-d interaction will be studied with crossing beams of polarized atoms from a polarized Atomic Beam Source (ABS) and polarized deuterons from a polarized ion source as it is shown in the scheme of Fig. 2 . The polarized ABS is based on the source from Ferrara University that was previously used in IUCF [10]. It delivers a beam of deuterium atoms of requested nuclear vector or tensor polarization and energies of about $0.1 \mathrm{eV}$. The intensity of the beam of $4 \cdot 10^{16}$ atoms/s, achieved at the IUCF, is sufficient for the measurements of the fusion reaction with low cross-section.

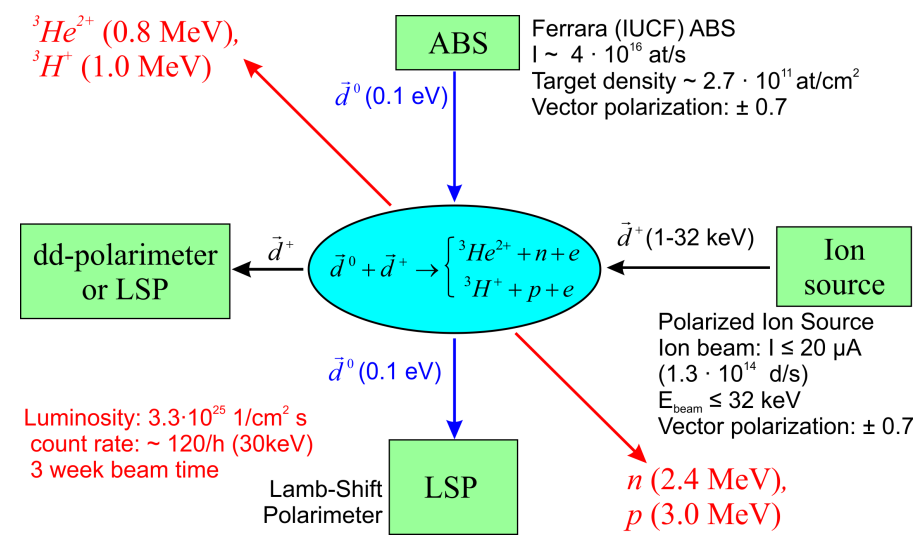

Figure 2: Layout of the experiment.

The atomic deuterium beam from the ABS is crossed by the beam of polarized deuterons from the POLarized Ion Source POLIS. This source is based on the injector equipment of the cyclotron at the Kernfysisch Versneller Instituut (KVI) of Rijksuniversiteit Groningen, The Netherlands. Nowadays the KVI scientific program has switched from light to the heavy ions and the light-ion source 
became available and is presently at our disposal. This source is capable of producing an ion beam of $20 \mathrm{~mA}$ and energies up to $35 \mathrm{keV}$. The current project requires a substantial upgrade of this source to increase the beam energy up to $100 \mathrm{keV}$. This energy is required to combine new experimental results with the existing data available for energies around $100 \mathrm{keV}$ and higher. Such an overlap is essential for a crosscheck with other data. A new ECR ionizer was designed, capable to increase the ion energy up to $100 \mathrm{keV}$. The use of the polarized atomic and ion beams requires the knowledge of their nuclear polarization. Instruments are necessary for the tuning of the polarized sources and for the constant monitoring as well. It is planned to use two polarimeters in the experiment, (i) a Lamb-shift polarimeter (LSP) and (ii) a nuclear-reaction polarimeter. The LSP allows more precise polarization measurements. More effort, however, is needed for its operation. Therefore, before the beginning of a data-production run it is used to tune first the ABS and the ion source. Then it is positioned as shown in Fig. 2 and is used to control the ABS-beam polarization from time to time in short measurements of $2 \mathrm{sec}$. The polarization of the ion beam is permanently monitored by the $\mathrm{d}$ - $\mathrm{d}$ polarimeter. It contains a 0.5 micrometer titanium foil with with unpolarized deuterium, implanted before the measurement by the ion beam. During the measurement, the angular distribution of the ejectiles from the $\mathrm{d}$ - $\mathrm{d}$ reaction is measured continuously with nine semiconductor detectors, positioned at appropriate angles around the titanium foil. One of the crucial tasks of the experiment is the detection of the fusion-reaction ejectiles, i.e., protons, tritium ions, and $3 \mathrm{He}$ ions (neutrons are not detected in this experiment). Typical count rates, aimed at, are 120 events per hour for $30 \mathrm{keV}$ ions. Therefore the detector system should have low background and noise to distinguish these rare events. A solution of this task should be simplified by two aspects:

- Due to the Q values, all reaction products have energies essentially higher than initial deuterons. These have an energy up to $100 \mathrm{keV}$ while the energy of the reaction products is in the range 0.8-3 MeV. Since the semiconductor detectors are energy-sensitive devices, they are used to identify the reaction products by their energy. Background caused by scattered electrons and deuterons can be discriminated against the ejectile spectra. The background contribution by the cosmic radiation and the radioactivity of the construction material has to be investigated.

- We investigate the two-particles reaction (two incident particles and two products) with well defined particle energies. Consequently, the particle identification by the energy will be reliable.

The main goal of the experiment is the measurement of the angular distributions of the reaction products. Therefore the detector system should cover the whole range of emission angles. Ideally it has to cover the $4 \pi$ solid angle around the d-d interaction volume with a typical angular resolution of 10-15 degree for a reliable comparison with theoretical calculations of the differential cross-section. A magnet system, providing the holding field for the polarization maintenance in the d-d interaction volume, was designed with permanent magnet elements. It provides a uniform magnetic field of the required strength (up to $320 \mathrm{G}$ ) in the beam interaction area. The direction of the magnetic field can be varied in wide range according to the experimental requirements. The detector system Fig. 3 was made as a cubic structure with the inner surface covered with 576 Hamamatsu S3590 silicon PIN diodes. The sides of the cube were produced by the printed-circuitboard technology and used as supporting structure for the diodes, and for the routing the detector 
signals as well. The sides of the detector wired to the connectors at the upper flange of the vacuum chamber. A special readout electronic module ASF48 was designed and produced for the data acquisition from the detector. Each module contains 48 charge-sensitive channels. The module provides continuous and simultaneous measurement in all 48 channels with six eight-channel pipeline ADC's. The signal in every channel is registered automatically in case of exceeding the amplitude trigger threshold and is sent to readout computer. Time of the signal is also recorded by the trigger actuation with $10 \mathrm{~ns}$ accuracy. Sampling frequency of the ADC is $65 \mathrm{MHz}$.

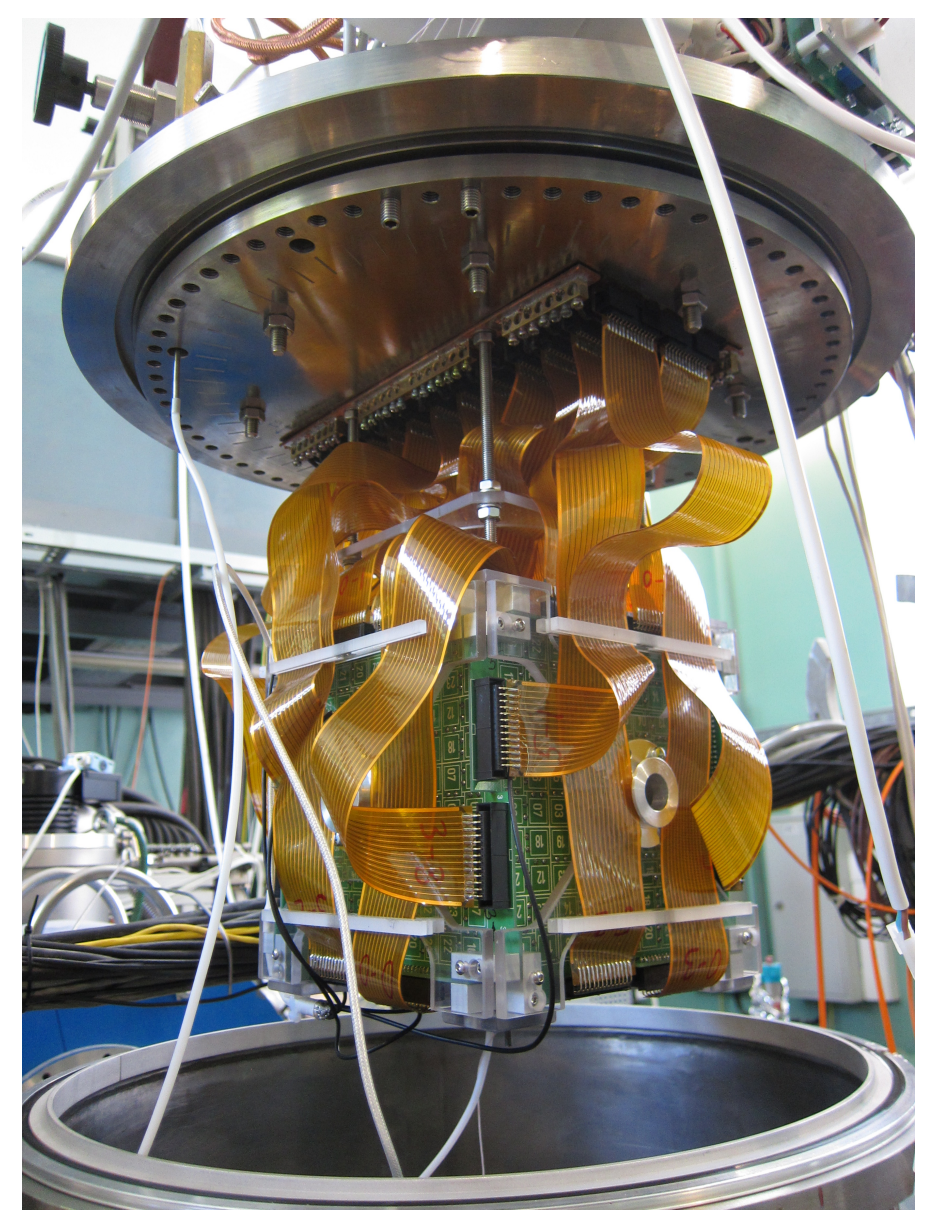

Figure 3: Detector system of the PolFusion experiment.

\section{Test measurement and software development}

An unpolarized experiment with ion beam and solid target was carried out, in order to check the detector system and the readout electronics. The target foil was made of deuterated polymethylmetacrylate and installed in the detector chamber at the outlet of the ion source, surrounded by a test setup of eight semiconducting detectors. The target was exposed to the ion beam of $10 \mathrm{~mA}$ and $15 \mathrm{keV}$ energy. The amplitude spectra obtained in this experiment show three distinct peaks of the deuterium fusion products, namely $0.8 \mathrm{MeV}^{3} \mathrm{He}^{2+}, 1 \mathrm{MeV}$ tritium, and $3 \mathrm{MeV}$ protons Fig. 4 . 
Successful registration of the d-d fusion reaction products confirms the selection of detector elements and the performance of the readout electronics. A mathematical apparatus was developed for experimental data analysis. It describes reactions of two particles with spin 1 in the initial state. Analytical formulae were obtained using partial wave expansion for the calculation of all observables of the reactions $\vec{d}+\vec{d} \rightarrow{ }^{3} \mathrm{He}+n$ and $\vec{d}+\vec{d} \rightarrow t+p$. The complete set of the matrix elements was evaluated in an explicit form for $\mathrm{S}, \mathrm{P}$ and $\mathrm{D}$ waves at low energies.

Analytical equations were developed for the d-d-reaction cross-section for any arbitrary polarization of the beam and the target, using mean values of the polarization operators of the density matrix of the initial state. This enables simulation of the experimental observables using the set of partial amplitudes.

Existing experimental data for the d-d fusion reaction at low energy were collected and analyzed, including the results of unpolarized and partially polarized measurements performed in the years 1956-2006 with solid and gas targets. A minimization algorithm was developed for the calculation of partial amplitudes from experimental data, making use of the collected data set and analytical functions obtained in partial wave expansion in complete spin of two-particle system representation.

A software is developed for calculation of the experimental observables. This software will be used for evaluation of full and differential cross-section, analyzing powers, correlation coefficients, and polarization transfer coefficients.

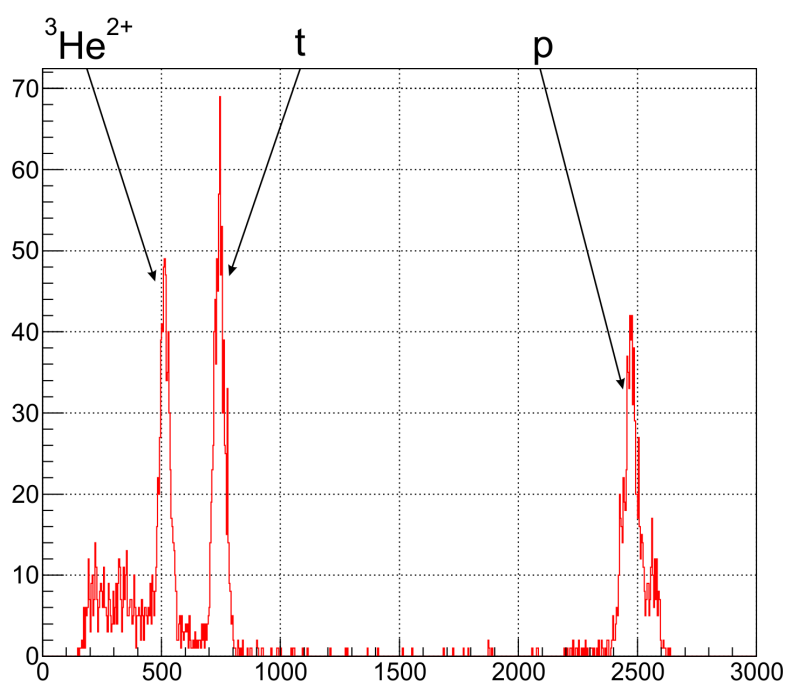

Figure 4: Typical ion spectra of first detector tests with unpolarized d-d fusion reactions at $15 \mathrm{keV}$. The protons $(3 \mathrm{MeV})$, tritons $(1 \mathrm{MeV})$ and ${ }^{3} \mathrm{He}^{2+}$ ions $(0.8 \mathrm{MeV})$ can be clearly identified with the PIN diodes.

\section{D-d and d-d fusion reaction}

With the present setup, the planned nuclear d-d fusion measurements are to be performed with the crossed polarized atomic D beam from the ABS (energy about $0.1 \mathrm{eV}$ ) and the polarized 20 to $100 \mathrm{keV}$ ionic $\mathrm{d}$ beam from the ion source from the ion. With decreasing center-of-mass energy, 
the electron-shielding effect is increasing. The non-zero probability to find the s electron in the range of the deuteron reduces the Coulomb repulsion between the deuterons. Therefore, in the D-d interaction at energies below $20 \mathrm{keV}$, the reaction cross section gets enlarged compared with that in the double-ionic d-d reaction encountered in a fusion reactor with highly ionized reaction partners. The electron screening effect, also important in astrophysical fusion, was confirmed with unpolarized deuterons [11] and is called the astrophysical S-factor. With the present setup and a future extension to store unpolarized or even polarized $\mathrm{D}$ atoms, the unpolarized data might be extended to single or even double polarized effects, including a polarized electron, with the use of the polarized ionic d beam of energies below $10 \mathrm{keV}$.

\section{References}

[1] R. M. Moon, T. Riste, and W. C. Koehler, Phys. Rev. A 181,920 (1969).

[2] T.E. Chupp et al., Nucl. Instr. and Meth. A 574,500 (2007).

[3] H. Paetz gen. Schieck, Eur. Phys. J. A 44,321 (2010).

[4] B.P. Adyasevich, D.E. Fomenko, Sov. J. Nucl. Phys. N9 167 (1969).

[5] B. Adjasevich, V. Antonenko. Measurements of the polarization correlation coefficients in reactions $d(d, p) t$ and $d(d, n)^{3} H e$. Preprint IEA-2704, Moscow (1976).

[6] R.M. Kulsrud, H.P. Furth, E.J. Valeo, M. Goldhaber, Phys. Rev. Lett. 491248 (1982).

[7] A. Honig, A. Sandorfi, Proceedings of the 17 International Spin Physics Symposium. AIP Conference Proceedings 9151010 (2007).

[8] J.-P. Didelez and C. Deutsch. Persistence of Polarization in a Fusion Process. EPJ Web of Conferences 304018 (2010).

[9] E. Epelbaum, Progr. Part. Nucl. Phys. 57654 (2006).

[10] T. Wise et al., Nucl. Instr. and Meth. A 336410 (1993).

[11] F. Raiola et al., Eur. Phys. J. A 13377 (2002). 